\title{
Structures Inside Living Cells Limit Optical Sectioning Precision
}

James Pawley

Zoology Department, University of Wisconsin, Madison, WI, 53706 USA

Confocal microscopy is inextricably linked to the concept of the optical section. Optical sectioning occurs because light originating from out-of-focus planes is excluded by the pinhole diaphragm in front of the photodetector. The idea of optical sectioning is so firmly embedded in the psyche of modern biological microscopy that few stop to ask whether the "plane-of-focus" is an actual geometrical plane similar to a mechanical section, or merely "the surface described by the array of points at which the laser beam reaches its best focus." This best focus plane will be something close to a geometrical plane only if the specimen is optically homogeneous (i.e., has a uniform refractive index: RI.). Although this is a fair approximation for embedded specimens, as more studies are performed on living cells, it is becoming clear that few living specimens are optically homogeneous. This should not surprise us: we have been viewing living cells using phase contrast and DIC for years. The contrast in these images reflects RI variations within cells.

The present work gives some indication of how serious this problem can be. Cheek cells were prepared fresh, stained with acridine orange and viewed in a 40-70 $\mu \mathrm{m}$ high chamber made by separating a coverslip from the slide with 4 dots of dried nail polish. This specimen was then viewed in a confocal microscope in the XZ plane while collecting both fluorescent and reflected light (Fig 1). Despite some signal loss with depth due to spherical aberration, the general features of the cells can be seen in both fluorescent and reflected light. Nuclei and features in the cortex outlining the cell margin are visible. However, the most noticeable feature in the right member of this pair is the tremendous difference in appearance between the reflection image of the near and far glass-water interfaces. While the image of the near (right) surface approximates a straight line, that of the far side (left) does not. The "dip" in it can be correlated with the presence of an overlying nucleus. Because we know that the surface of the slide is actually flat, the fact that its image is not a line indicates that the optical section is not flat under refractile features such as nuclei. Measurements from this data, show that the nucleus has displaced the "best focus plane" downwards by $\sim 6 \mu \mathrm{m}$ for all planes below it. Figure 2 diagrams the cause of this distortion. Figure 3 is a stereopair projection image of a similar specimen showing the same effect as Fig.1.

Perhaps these images explain the common observation that, on a confocal microscope, one seldom records small fluorescent features on the far side of a nucleus in a living cell. As living cells necessarily have nuclei and other refractive organelles, it is not clear how we can avoid the sequellae of their optical properties. In some cells these features are naturally less optically disruptive: cornea, lens and retina come to mind. For other tissues, it would be well to remember that the visibility of peri-nuclear structures may depend strongly on whether or not they are located on the side nearest to the objective. When looking far below the surface of a tissue, it may be useful to try to arrange matters so the foreground contains as few nuclei as possible. 


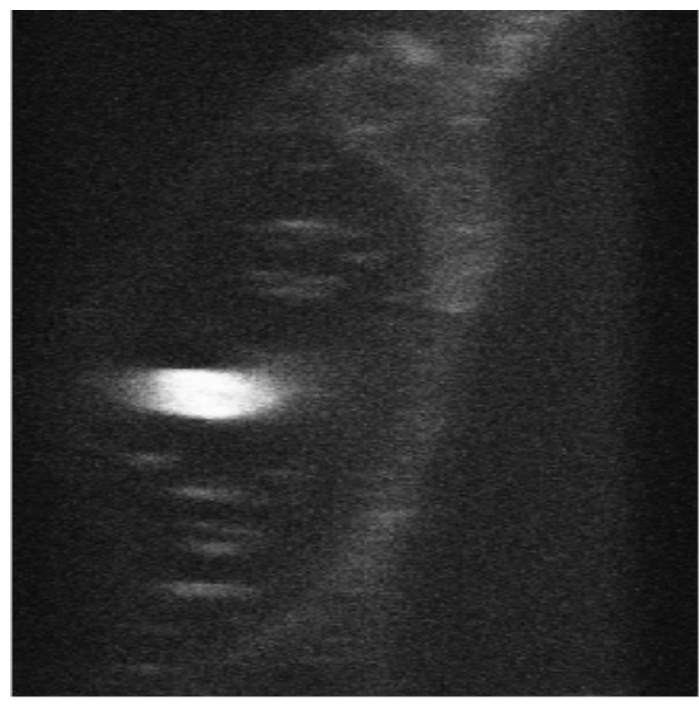

Acridine Orange fluorescence

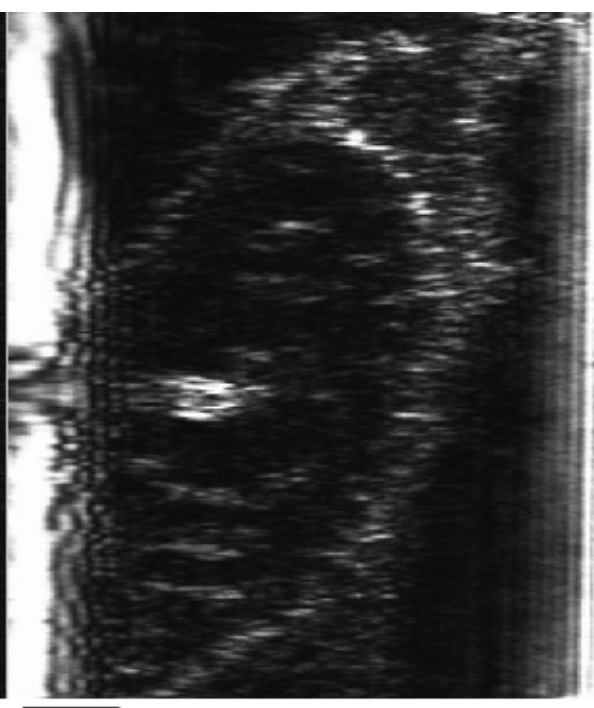

Backscattered light

X-Z images, Cheek cells in saline, Nikon $60 \times 1.4$ PlanApo Oil, Radiance-Plus

Figure 1

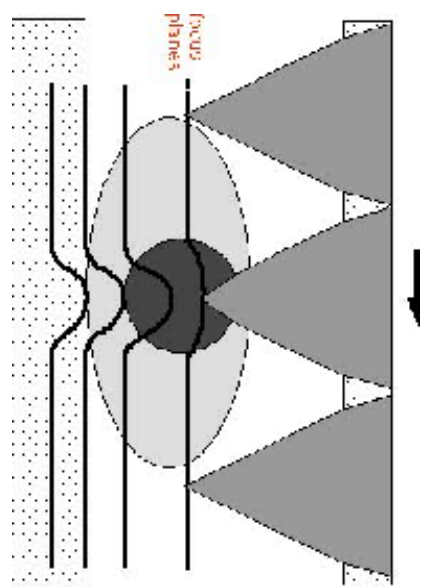

Figure 2: Schematic diagram of how the presence of a spherical nucleus of higher RI could focus plane of a light beam (right) scanned over it.

All figures oriented so that the objective is to the right. Cross eyes to view stereo pair.

<jbpawley@facstaff.wisc.edu>
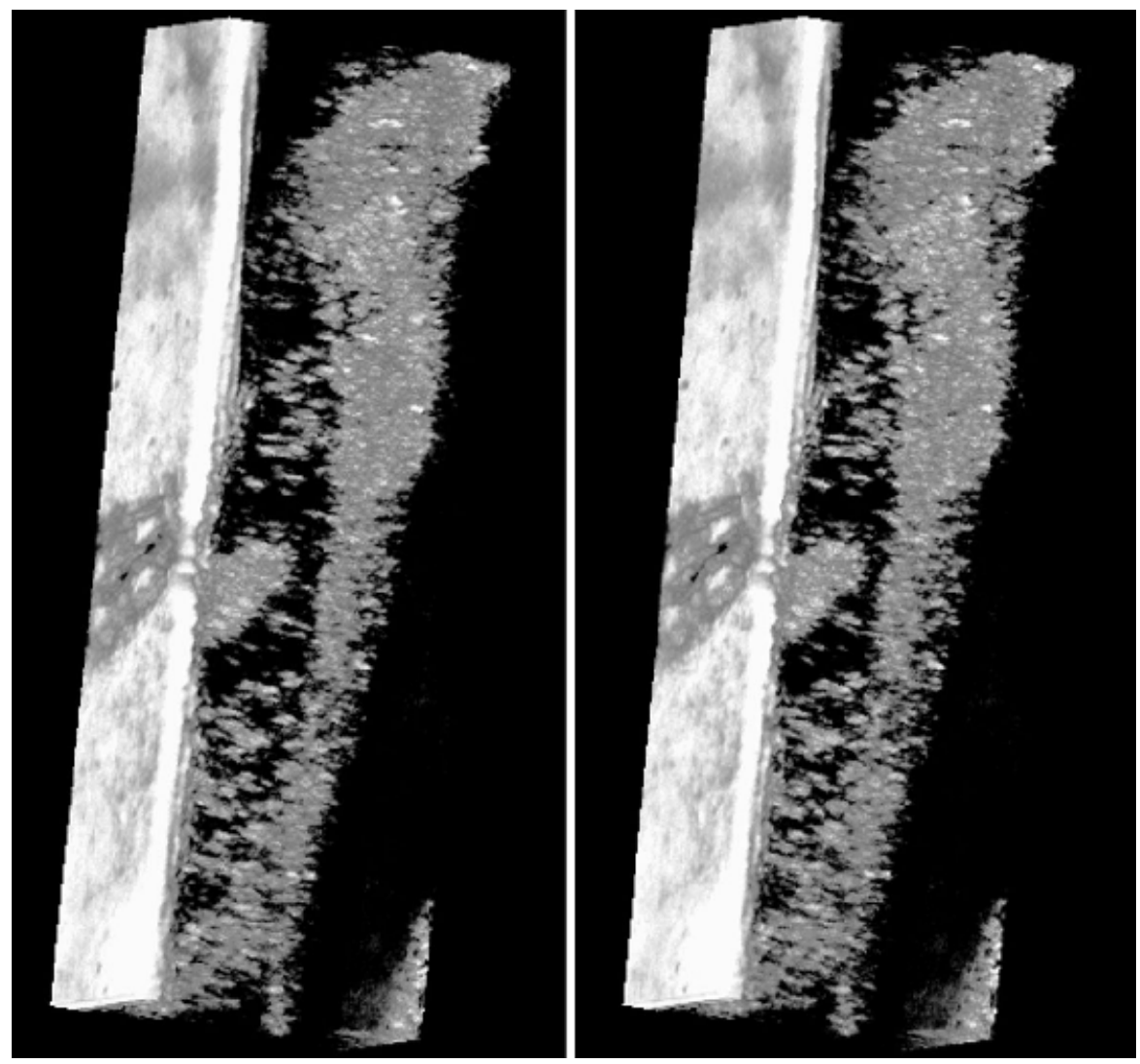

Figure 3: Stereopair looking up from the far side of a 5122x512 x169 BSL data set of a cheek cell. The optical section distortion produced by the nucleus is evident as the "shadow" image of the nucleus on what should be a featureless, white image of the slide surface 